\title{
Fístula perilinfática traumática: Entidad otorrinolaringológica poco frecuente
}

\section{Traumatic perilymphatic fistula : A rare otorhinolaryngological entity}

\author{
Carlos Stott $C^{1}$, Patricio Tabilo $C^{1}$, Nicolás Albertz $A^{2}$, Cesar Toro $A^{1}$.
}

\section{RESUMEN}

La fístula perilinfática corresponde a una patología infrecuente en la práctica otorrinolaringológica diaria Esta puede tener un origen congénito, de aparición espontánea o más frecuentemente postraumática, presentando habitualmente la triada clínica de hipoacusia, tinnitus y vértigo. A continuación se presenta el caso clínico de una paciente de 50 años atendida en el Servicio de Atorrinolaringología del Hospital Cínico de la Universidad de Chile, ingresada por un cuadro con clínica compatible, de inicio súbito luego de una perforación timpánica traumática autoinferida.

Palabras clave: Fistula perilinfática, postraumática, cirugía de estribo, hipoacusia.

\section{ABSTRACT}

Perilymphatic fistula is an infrequent pathology in standard otorhinolaryngological practice. Fistulas of this sort may be of congenital, spontaneous or, more frequently, post traumatic origin, normally presenting with the clinical triad of hearing loss, tinnitus and vertigo. The case of a 50 year old patient presenting with clinically compatible symptoms of sudden appearance after a self-infringed traumatic tympanic perforation is discussed.

Keywords: Perilymphatic fistula, post traumatic, stapedius surgery, hearing loss.

\section{INTRODUCCIÓN}

La fístula perilinfática corresponde a una comunicación anormal entre el oído interno y oído medio 0 mastoides, secundario a un defecto óseo en la cápsula ótica, ventana oval o ventana redonda. Esta se ha descrito en el contexto traumático, de aparición espontánea o congénita, asociada con malformaciones de oído medio ${ }^{1,2}$, teniendo todas ellas la presencia de una alteración anatómica particular. De esta manera la dehiscencia ósea debe estar presente de manera imprescindible para hacer el diagnóstico.

Asimismo se describe muchas veces a la fístula perilinfática como resultado de complicaciones de cirugías del estribo ${ }^{3,4}$, debiéndose a una falta de cierre de la ventana oval, o por fracturas o fisuras de la platina del estribo causadas por maniobras inapropiadas sobre éste. Se presenta con una clínica caracterizada por vértigo objetivo e hipoacusia sensorioneural que en muchos casos

\footnotetext{
1 Médico, Servicio Otorrinolaringología y Cirugía de Cabeza y Cuello, Hospital Clinico Universidad de Chile.

2 Interno de Medicina, Facultad de Medicina, Universidad de Chile.
} 
puede ser incluso cofosis súbita con una patogenia poco clara $^{5}$, que se suele acompañar de tinnitus.

Pese a ser una patología poco frecuente, la fístula perilinfática asociada a traumatismos de oído tiene que ser sospechada y debe ser descartada ante una historia clínica sugerente, dada la importancia que reviste el manejo precoz para evitar las secuelas, sobre todo de la esfera auditiva a largo plazo que conlleva la alteración estructural subyacente.

En este sentido se han intentado diferentes esfuerzos para disminuir los efectos deletéreos secundarios a la disrupción anatómica de la unión entre el oído medio e interno, utilizando terapias combinadas de antibióticos tópicos o sistémicos, 0 bien corticoides, terapias no exentas de controversia y discutida efectividad. Sin embargo el único tratamiento que ha demostrado resultados clínicos satisfactorios corresponde a la cirugía precoz, razón por la que se insiste en el diagnóstico oportuno y la derivación al especialista competente.

\section{CASO CLÍNICO}

Se presenta el caso de una paciente de 50 años, de sexo femenino, sin antecedentes mórbidos de importancia que consulta en el servicio de urgencia por cuadro caracterizado por vértigo de tipo objetivo asociado a importante sintomatología neurovegetativa con náuseas y vómitos profusos, acompañado de tinnitus permanente e hipoacusia de oído derecho. El cuadro se inicia súbitamente luego que la paciente realizara maniobras para disminuir su prurito ótico con un palillo de madera largo de cóctel, que resulta en una perforación timpánica luego de realizar un movimiento brusco con el objeto. Es derivada a otorrinolaringología con el diagnóstico de perforación timpánica secundaria a trauma, indicándose tratamiento con antivertiginosos y reposo hasta hacer efectiva la atención por especialista. Por persistencia del cuadro la paciente consulta en el servicio de otorrinolaringología luego de 5 días de ocurrido el evento traumático.

Al examen físico otorrinolaringológico de ingreso destaca la presencia de nistagmus espontáneo grado III con fase rápida a derecha el cual disminuye con la fijación ocular. En la otoscopía se evidencia una perforación timpánica en el cuadrante posteroinferior de aspecto traumático en el oído derecho, destacando en la prueba de diapasones de Weber lateralización a izquierda y Rinne (-) en oído derecho. El signo de la fístula en este caso resultó ser negativo, sin otros hallazgos significativos al examen físico. Con este cuadro clínico se decidió la hospitalización con el diagnóstico de perforación timpánica traumática y sospecha de fístula perilinfática secundaria de oído derecho.

En su estudio destaca una audiometría de tonos puros (Figura 1) que evidencia una anacusia del oído afectado, que además informa del tinnitus permanente referido por la paciente, con oído contralateral indemne. No fue posible realizar la prueba de VIII par dada la intensa sintomatología neurovegetativa presente en la paciente.

La imagenología con tomografía computada de oídos (Figura 2) evidenció la pérdida de continuidad y alteración anatómica concordante con el diagnóstico de fístula perilinfática traumática que posteriormente fue reconstruida en imagen $3 \mathrm{D}$ con el software Osirix ${ }^{\circledR}$ para dar una imagen dinámica preoperatoria

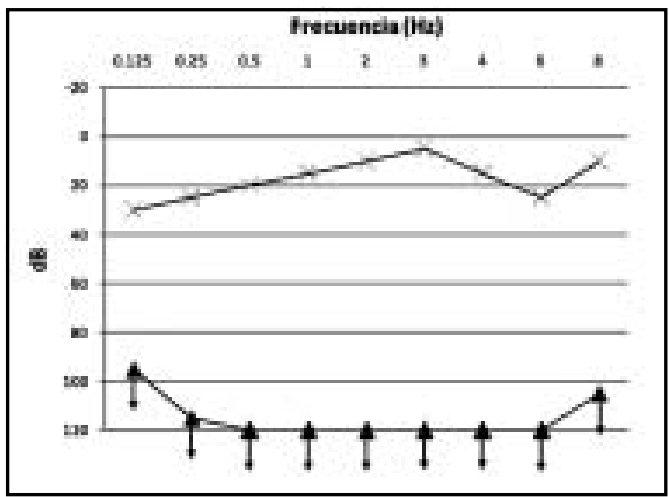

Figura 1.

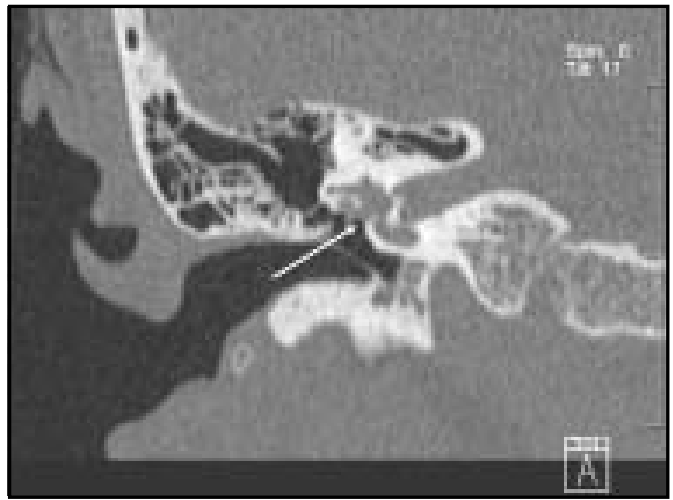

Figura 2. En la figura se aprecia la zona de la ventana oval con un velamiento que corresponde a la zona de hundimiento del estribo en la ventana oval y un tejido de inflamación sobre ésta. 
de certeza que apoyó y confirmó el diagnóstico previo a la resolución quirúrgica (Figura 3).

Desde su ingreso se inicia tratamiento antibiótico sistémico y ótico tópico más corticoides por vía oral previo a su resolución quirúrgica, evolucionando con discreta mejoría clínica con menos vértigo objetivo y disminución de nistagmus (grado II), ingresando a pabellón para timpanotomía exploradora, la cual se hizo efectiva al sexto día postrauma.

En la cirugía se observa una perforación timpánica lineal de aproximadamente $3 \mathrm{mms}$ en el cuadrante posteroinferior, la mucosa del promontorio y rodeando la ventana oval destaca engrosada dando salida a perilinfa de moderada cuantía. Además destaca la presencia de una luxación posteroinferior del estribo con hundimiento de la platina sobre la ventana oval que se corrige y reduce utilizando injerto de pericondrio de trago cubriendo la ventana oval, disminuyendo considerablemente la salida de perilinfa.

En su evolución postoperatoria la paciente permanece con nistagmus grado II sin embargo refiere menos vértigo pero con persistencia de anacusia en oído derecho sin nueva sintomatología acompañante. En controles posoperatorios a las 4 y 8 semanas la paciente refiere crisis vertiginosas de manera ocasional, sin embargo con persistencia de la pérdida auditiva.

\section{DISCUSIÓN}

Las fístulas perilinfáticas postraumáticas han sido descritas luego de barotrauma, daño ótico, daño en hueso temporal y particularmente fractura de éste ${ }^{6}$. Se localizan en las ventanas oval y redonda ya que corresponden a zonas débiles de la cápsula ótica, produciéndose pequeños defectos en ellas provocando pérdida de perilinfa desde oído interno hacia el oído medio.

La sintomatología presente en las fístulas perilinfáticas postraumáticas puede aparecer de forma inmediata dependiendo del tipo de trauma en cuestión, presentando una clínica evidente 0 bien sólo síntomas menores e intermitentes que se desencadenen por cambios de presión del oído medio. Es así como se presentan crisis vertiginosas asociado a nistagmus horizonto-rotatorio y síntomas neurovegetativos importantes. El estudio vestibular con prueba calórica revela hasta $50 \%$ de alteraciones.

El signo de Hennebert, descrito inicialmente en pacientes con sífilis congénita, consiste en aplicar presión sobre el conducto auditivo externo movilizando mínimamente la membrana timpánica, produciendo de esta forma síntomas y signos vestibulares, el cual se puede ver hasta en el $40 \%$ de estos pacientes ${ }^{3}$. Asimismo se puede observar el signo de la fístula que aparece al aplicar variaciones de presión en el oído externo con otoscopio neumático o bien mediante la presión digital sobre el trago produciendo desviación conjugada de la mirada en la fase de la presión negativa. Este signo fue considerado patognomónico, sin embargo posteriormente se ha demostrado que su sensibilidad y especificidad son bajas, presentando hasta $40 \%$

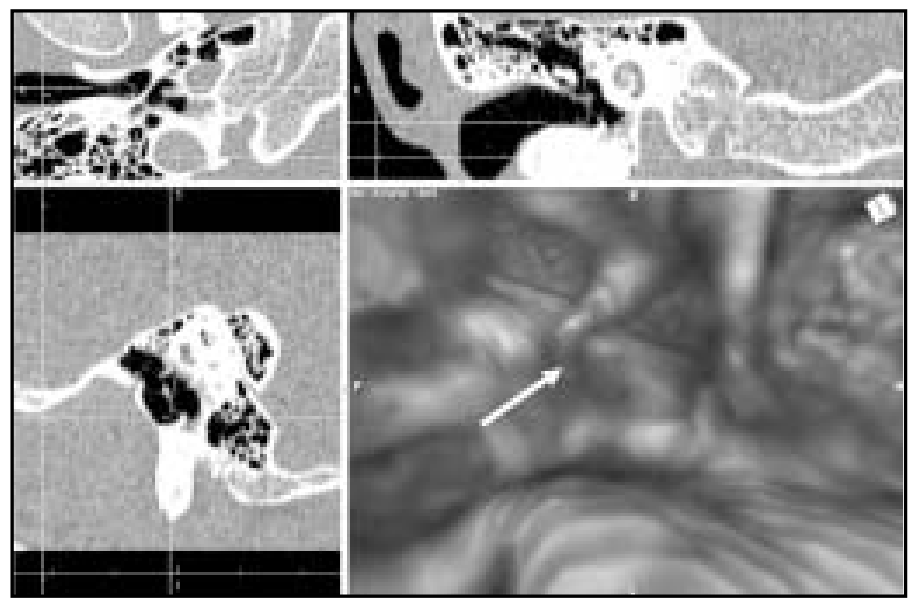

Figura 3. En esta figura de una reconstrucción se aprecia el hundimiento del estribo en la ventana oval y la subluxación del yunque 
de falsos positivos. También se puede encontrar el fenómeno de Tullio que consiste en una crisis de vértigo y nistagmus desencadenada por un estímulo sonoro de alta intensidad que se relaciona con una solución de continuidad ósea 0 membranosa. Dentro de las causas conocidas de dicho fenómeno están además de la fístula perilinfática, la dehiscencia del canal semicircular superior y la sífilis congénita, esta última a consecuencia de la hiperlaxitud del ligamento anular de la platina del estribo $0^{7,8}$.

Al examen físico además se evidencia una prueba de Romberg que lateraliza hacia el lado afectado acentuándose al perder la fijación ocular que revela la causa periférica del vértigo. La otoscopía puede evidenciar hemotímpano 0 bien mostrar una pequeña perforación puntiforme 0 lineal como en el caso clínico analizado. En ciertos casos también se puede encontrar un tímpano completamente sano.

Frente a la sospecha de la alteración anatómica particular, estos signos se buscan dirigidamente en la exploración otorrinolaringológica, estando muchas veces ausentes pese a la presencia de enfermedad, motivo por el cual estos hallazgos deben ser considerados sólo como elementos de orientación, sin embargo ninguno de ellos es patognomónico quedando el diagnóstico en evidencia en el acto quirúrgico. Por este motivo resulta importante recalcar que el clínico se debe guiar no sólo por la presencia o ausencia de estos hallazgos, sino que más importante es la historia clínica sugerente y examen físico compatible, que son los elementos más importantes en el diagnóstico de fístula perilinfática traumática9 .

En la audiometría de tonos puros se puede apreciar hipoacusia sensorioneural o mixta dependiendo si además de la fístula existió daño de la cadena osicular. Puede ser fluctuante 0 súbita, y aunque no es lo habitual también puede existir normalidad auditiva. Asimismo el tinnitus aparece en la mayoría de los casos.

Dado que en el acto quirúrgico no siempre es posible evidenciar la pérdida de perilinfa se han desarrollado exámenes complementarios tendientes a un mayor acercamiento diagnóstico. Por ejemplo tenemos el caso de la fluoresceína intratecal o intravenosa, la cual se ha demostrado que puede ser detectada en la perilinfa y podría ser útil en la detección de fístulas perilinfáticas ${ }^{10}$. Por otro lado está el caso de las fístulas perilinfáticas congénitas, en las cuales exámenes como la ß-2 transferrina, sustancia presente en el líquido cefalorraquídeo y perilinfa, detectadas por estudios electroforéticos y de inmunoblotting, orientan hacia la existencia de la alteración anatómica ${ }^{11,12}$ en caso de estar presente. Desafortunadamente estas pruebas no detectan de manera satisfactoria los casos de fístula perilinfática lo que hace que sean pruebas poco confiables.

Pese a toda la orientación clínica que debe tener un alto índice de sospecha el diagnóstico no es fácil, no existiendo una prueba diagnóstica única patognomónica, siendo éste muchas veces controversia $\left.\right|^{13}$. Así a través de la historia clínica y examen físico se debe establecer un diagnóstico presuntivo, sin embargo el examen de mayor rendimiento lo tiene la tomografía computada, que tiene alto rendimiento en detectar lesiones óseas, y que además permite realizar reconstrucciones dinámicas preoperatorias. Pese a esto el diagnóstico de certeza se realizará de manera intraoperatoria con la observación de la salida de perilinfa ${ }^{3}$.

Respecto de las medidas terapéuticas estudios previos han concluido que la cirugía exploradora a través de timpanotomía está fuertemente indicada en pacientes que sufren de trauma del hueso temporal o trauma penetrante de oído medio que demuestren persistencia de síntomas vestibulares, hipoacusia sensorioneural o evidencia imagenológica de patología de ventana ${ }^{6}$. Sin embargo el manejo conservador estará indicado en los casos de sintomatología leve y estacionaria.

Finalmente en lo que a pronóstico se refiere éste es un tanto incierto desapareciendo en muchos casos la sintomatología al cabo de 4 a 6 semanas, sin embargo ésta puede mantenerse hasta después de varios meses, sobre todo la pérdida auditiva que puede ser permanente.

\section{BIBLIOGRAFÍA}

1. Vignaud J, Marsot-Dupuch K, Pharaboz C, Derosier C, CordolianI Y. Imaging of The Vestibule. Oolaryngol Head Neck Surg 1995; 12: 36-49.

2. Nakashimaa T, Sonea M, Teranishia M, TominagaA $M$, Sugiuraa $M$, Naganawa $S$. Imaging Of $A$ 
Congenital Perilymphatic Fistula. Int J Pediatr Otorhinolaryngol 2003; 67: 421-5.

3. García de HA, Pérez A, Viada J, Cadaval F. Meningoencefalitis Post Estapedotomía. Análisis de un Caso Clínico. Rev Méd Chile 2004; 132: 1407-11.

4. Stott C, Inzunza F, Walker K, Bahamonde $\mathrm{H}$. Timpanoplastía en Empalizada. Rev Corrinolaringol Cir Cabeza Cuello 2002; 62: 238-42.

5. Foster PK, Luebke AE. A Model For Perilymphatic Fistula Induced Hearing Loss In The Guinea Pig Cochlea. Hear Res 2002; 167: 175-9.

6. Soo KH, Kazahaya K, Handler SD. Traumatic Perilymphatic Fistulas In Children: Etiology, Diagnosis And Management. Int $\mathrm{J}$ Pediatr Otorhinolaryngol 2001; 60: 147-53.

7. Montojo J, Heitzmann T, Rubio L. Dehiscencia Del Conducto Semicircular Superior: Presentación De Un Caso Y Revisión Bibliográfica. Acta Corrinolaringol Esp 2005; 55: 171-5.
8. Esquivel P, ZúñIga J. Síndrome de Dehiscencia del Canal Semicircular Superior. Rev Aorrinolaringol Cir Cabeza Quello 2005; 65: 233-40.

9. Lo S, Mehta D, Daudia A. Perilymph fistula-a complication of penetrating injury to the ear not to be missed. Injury, Int. J. Care Injured 2003; 34: 238-9.

10. Poe DS, Gadre aK, Rebeiz eE, Pankratov mM. Intravenous fluorescein for detection of perilymphatic fistulas. Am J Ood 1993; 14: 51-5.

11. Weber PC, Kelly RH, Bluestone CD, Bassiouny M. Beta 2-transferrin confirms perilymphatic fistula in children. Otolaryngol Head Neck Surg 1994; 110: 381-6.

12. Wall C, Rauch SD. Perilymph fistula pathophysiology. Otolaryngol Head Neck Surg 1995; 112: 145-53.

13. Weber PC, Bluestone CD, Pérez B. Outcome of Hearing and Vertigo After Surgery for Congenital Perilymphatic Fistula in Children. Am J Colaryngol 2003; 24: 138-42. 